\title{
PHOTOCHEMICAL REACTIONS OF SILSESQUIOXANE-BASED RESISTS FOR ArF EXCIMER-LASER LITHOGRAPHY
}

\author{
Taku MORISAWA, Hiroshi FUKUDA, and Hiroshi SHIRAISHI \\ Central Research Laboratory, Hitachi Ltd. \\ Kokubunji, Tokyo 185, Japan
}

\begin{abstract}
Two silsesquioxane polymers were investigated as $\mathrm{ArF}$ excimer-laser resist materials. Polyphenylmethyl silsesquioxane (PMSQ) showed positive tone characteristics when an aqueous base developer was used, while polyhydroxybenzyl silsesquioxane (HSQ) showed negative tone characteristics. Infrared (IR) spectra and X-ray photo-electron spectrometry (XPS) indicate that a major imaging mechanism is the polarity change in PMSQ. Both materials showed sub-0.2- $\mu \mathrm{m}$ resolution capability for ArF excimer laser exposure with aqueous base development.
\end{abstract}

\section{Introduction}

ArF excimer-laser lithography is one of the most promising optical lithography technologies after $\mathrm{KrF}$ excimer-laser lithography. However, conventional phenolic polymer-based resist cannot be used as single layer resists at this wavelength (193 $\mathrm{nm})$ due to the extremely high photo-absorption of their phenyl ring structure. To solve this problem, single layer chemically amplified resists based on transparent methacrylate polymers have been intensively investigated $[1,2,3]$. Top surface imaging systems and bilayer resist systems have also been introduced to avoid some of the limitations of single layer resist processes, such as substrate reflection and resist pattern collapse $[4,5,6]$. Furthermore, an all-dry photolithographic process utilizing plasma-polymerized organo-polysilane has been introduced for ArF excimer-laser lithography [7].

We have recently shown that polyhydroxybenzyl silsesquioxane (HSQ) acts as a high resolution negative tone resist for ArF excimer laser exposure and that the obtained patterns can be used as a mask in etching underlying polysilicon films [8]. 
This suggests the possibility of a new process, shown in Fig. 1, which we call direct formation of hard-masks process. In this process, a directly patterned $\mathrm{SiO}_{2}$-like thin resist film is used as a hard-mask for dry-etching metals or silicon. Except for the film thickness, this very simple process resembles conventional single-layer resist process. It is therefore likely to represent a cost-effective alternative to more complex proposed process.

Silsesquioxane polymers are attractive for resist materials using this process because of their $\mathrm{SiO}_{2}$-like structure and their spin-coatable and aqueous base soluble characteristics $[9,10]$. We have investigated photo-reactions for various silsesquioxane polymers under ArF irradiation. In our previous work, however, excessively high photo-absorption in HSQ limited the photo-sensitivity and the possible resist thickness.

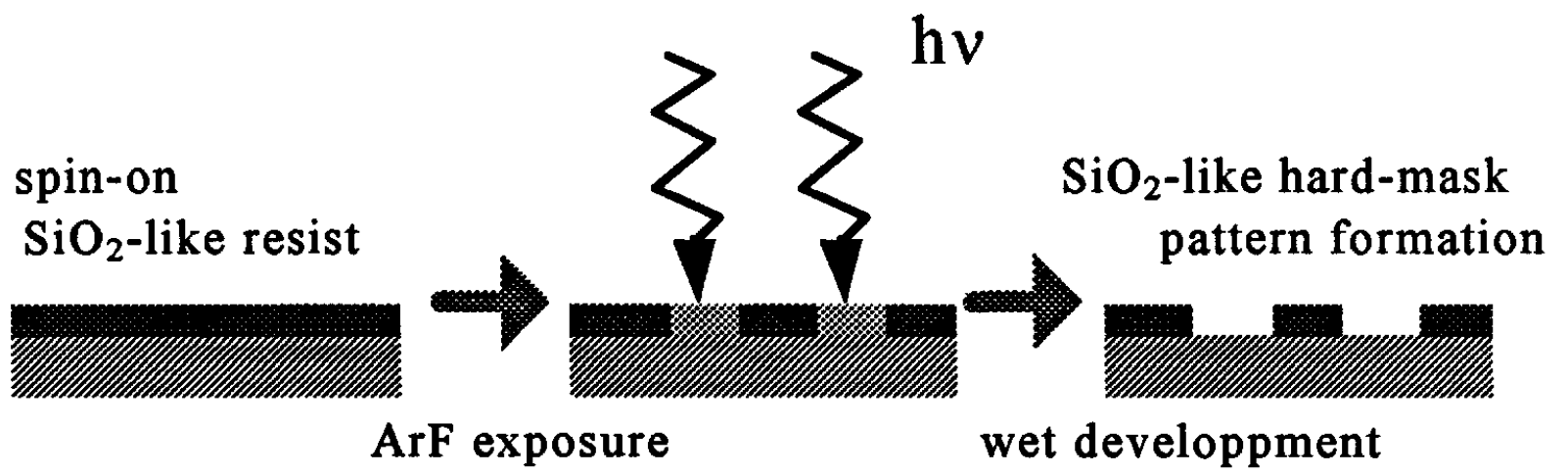

Figure 1 Process scheme of direct hard-mask formation.

In this paper, we describe a new ArF excimer-laser irradiation-induced photochemical reaction in which a positive-tone image is obtained using standard aqueous-base developer. We discuss the positive working mechanisms of polyphenylmethyl silsesquioxane, compared with the previously reported negative working mechanisms of HSQ.

\section{Experimental}

Polyphenylmethyl silsesquioxane (PMSQ) was obtained from Showa Denko Co. under the trade name GR-100 and polyhydroxybenzyl silsesquioxane (HSQ) was supplied by Hitachi Chemical Co.. Their chemical structures are shown in Fig. 2. The PMSQ and HSQ coating solvents were, respectively, propylene glycol 1monomethylether 2-acetate and ethyl cellosolve acetate. A commercially available aqueous base developer NMD-3 (tetramethylammonium hydroxide (TMAH), Tokyo Ohka Co.) was used as the developer. 
Exposure characteristics were obtained by using an ArF excimer laser (EMG 201 MSC, Lambda Physik). Sample films were coated onto silicon wafers (thickness: $50-100 \mathrm{~nm}$ ), and baked at $90^{\circ} \mathrm{C}$ for $180 \mathrm{~s}$ on a hot plate. The film thickness was determined with an Alpha Step-200 profilometer (Tencor Inst.). Infrared (IR) spectra were measured with a Fourier-transform IR spectrometer, JEOL JIR-100. X-ray photoelectron spectrometry (XPS) was performed for the PMSQ films before and after ArF laser exposure with a Shimazu-KRATOS XSAM800.

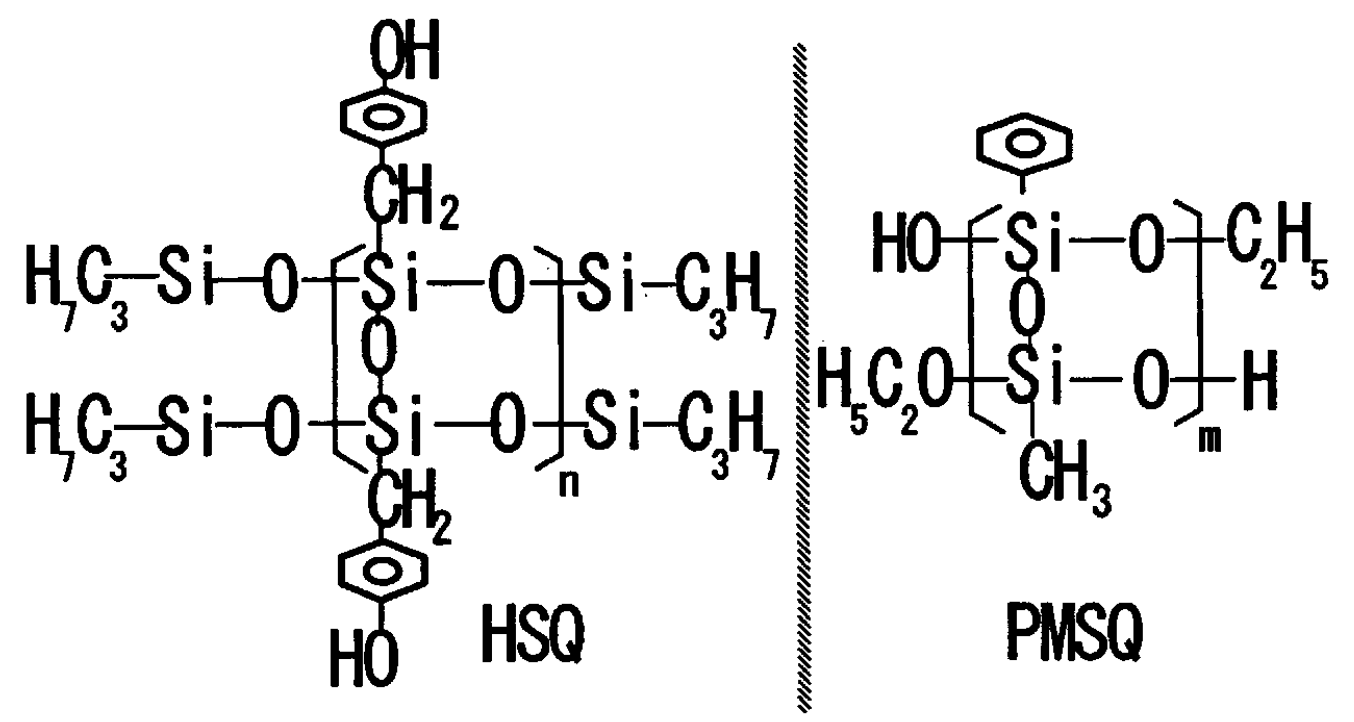

Figure 2 Chemical structures of polyhydroxybenzyl silsesquioxane (HSQ) and polyphenylmethyl silsesquioxane (PMSQ).

\section{Results and discussion}

\subsection{Basic characteristics}

We have examined various kinds of silsesquioxane polymers as resists for the direct formation of hard-masks process. Since the phenyl ring shows strong absorption at $193 \mathrm{~nm}$, the phenyl ring structure itself can be a photo-active group in the resist materials. Exposure characteristics of two examples (PMSQ and HSQ) are shown in Fig. 3. As shown in the figure, HSQ acts as a negative resist, whereas PMSQ acts as a positive resist when aqueous-base development is used. PMSQ is insoluble in the aqueous-base developer, however, and the ArF exposed area became aqueous-base soluble by NMD-3 $2.38 \mathrm{wt}$. \% at doses above $\sim 100 \mathrm{~mJ} / \mathrm{cm}^{2}$. 
The observed contrast of PMSQ was promising, as shown in the figure. HSQ is aqueous-base soluble due to the phenolic moiety at the side chain of its ladder siloxane structure. ArF irradiation can destroy the phenolic structure and form siloxane networks to cause aqueous-base insolubility in the exposed area.

\subsection{IR and XPS analysis}

To investigate the positive working nature of PMSQ, IR spectra of films before and after exposure were measured (Fig. 4). The silanol group is known to be an aqueous base soluble moiety [11]. As can be seen in Fig. 4, ArF excimer-laser irradiation resulted in an increase of the silanol moiety in the PMSQ film. However, the absorption spectra due to the silanol group showed a small increase at the dose of $600 \mathrm{~mJ} / \mathrm{cm}^{2}$, at which a positive tone had already been obtained.

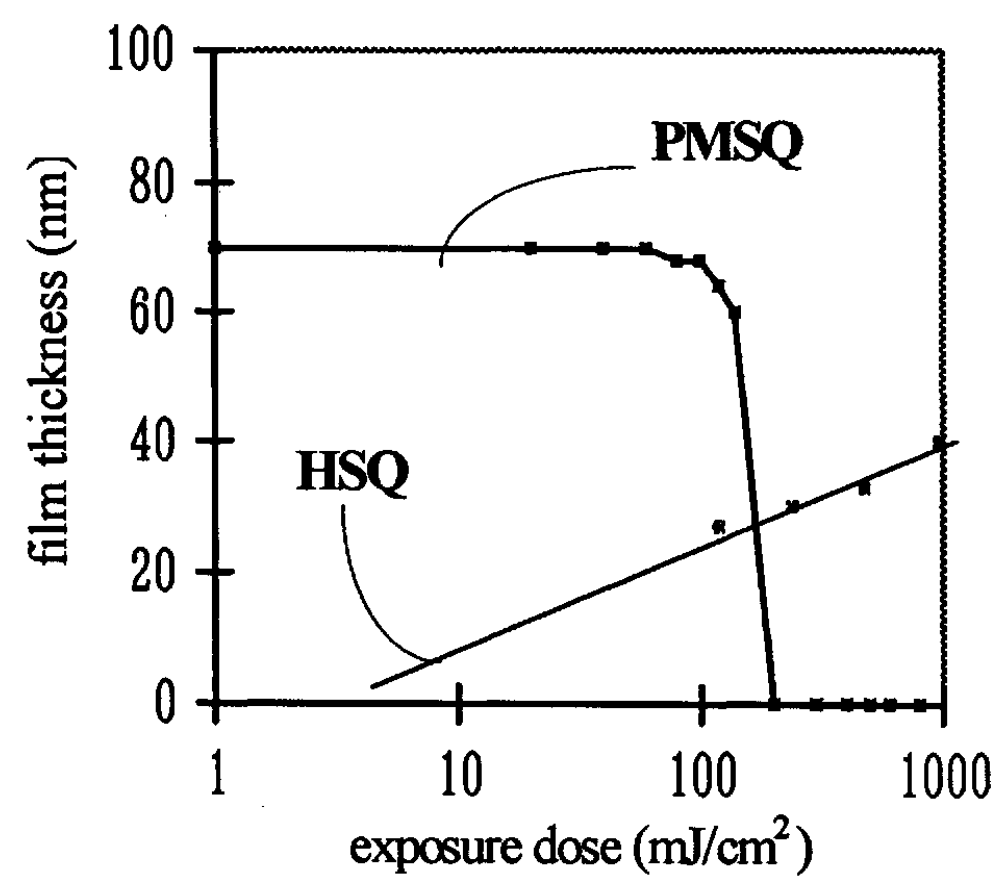

Figure 3 Exposure characteristics of HSQ and PMSQ initial film thickness, HSQ: 50-nm, PMSQ: 70-nm, development time: $45 \mathrm{~s}$ 


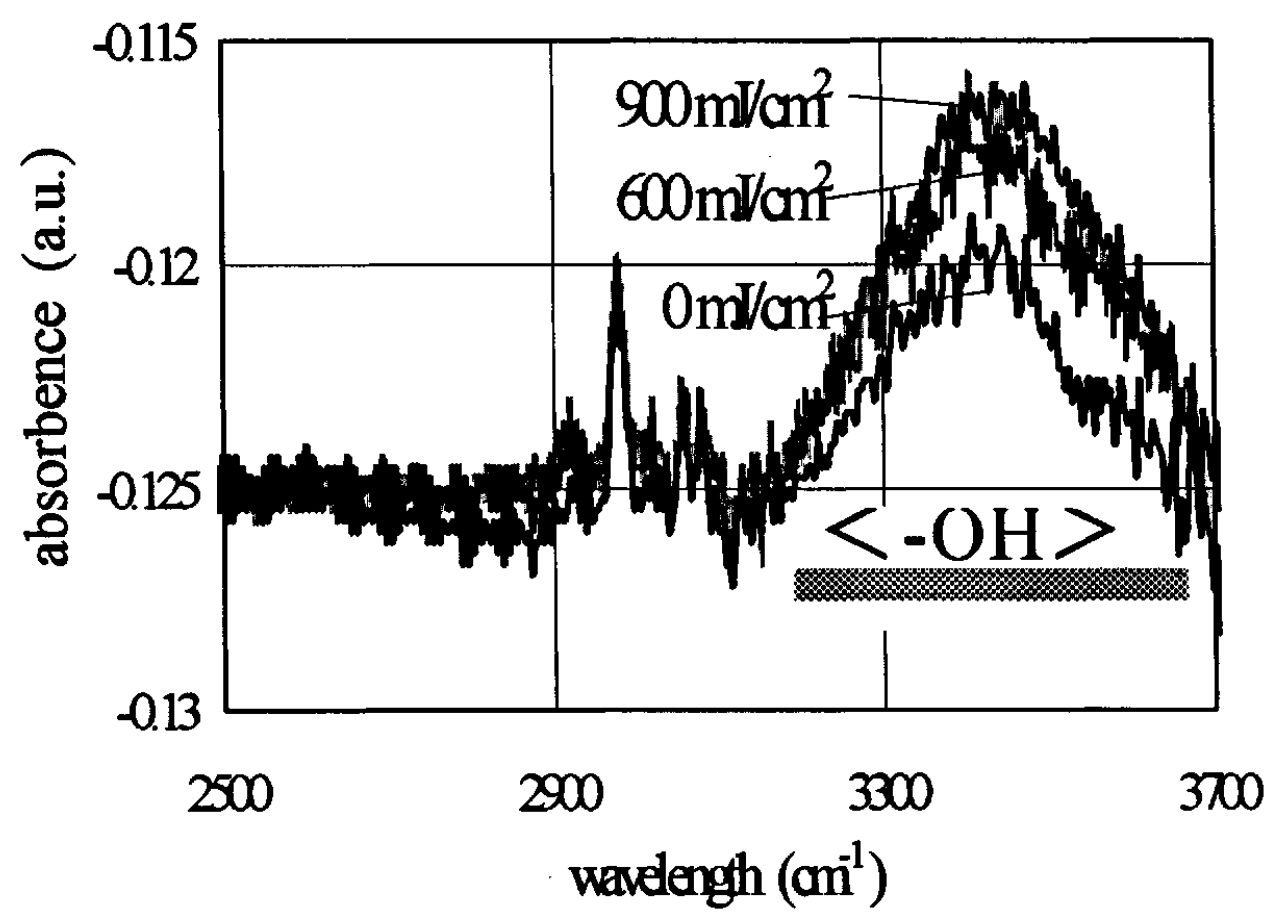

Figure 4 IR spectra of PMSQ films before and after ArF excimer laser exposure.

To evaluate the degree of photo-oxidation, we applied XPS analysis to PMSQ before and after the ArF exposure at $100 \mathrm{~mJ} / \mathrm{cm}^{2}$. The results are summarized in Table 1. The atomic ratio of oxygen to silicon in PMSQ films was 1.7 before exposure, and became about 2.0, the same as silicon dioxide, after exposure. Therefore, photo-oxidation induced by ArF excimer-laser irradiation proceeds effectively even at a dose of $100 \mathrm{~mJ} / \mathrm{cm}^{2}$.

Table 1. Elemental fraction of PMSQ before and after ArF excimer laser exposure.

\begin{tabular}{|c|c|c|c|}
\hline atomic ratio & $\mathrm{Si}$ & $\mathrm{O}$ & $\mathrm{C}$ \\
\hline before exposure & 16.6 & 27.2 & 56.3 \\
\hline after exposure & 17.4 & 35.2 & 47.5 \\
\hline
\end{tabular}

exposure dose: $100 \mathrm{~mJ} / \mathrm{cm}^{2}$

\subsection{Chemical reaction}

The aqueous-base solubility of resists depends not only on the concentration of base-soluble moieties but also on their orientation or positions in the resist matrix[12]. Though the PMSQ film was not soluble in standard aqueous-base developer, as we explained, it did have some silanol before exposure, as shown in 
Fig. 4, and was soluble in a stronger aqueous base solution with a TMAH concentration of over $3 \mathrm{wt}$. \%. From these results, the following dissolution inhibition and promotion mechanism of PMSQ is proposed. The PMSQ film is potentially soluble in an aqueous-base solution since it contains hydrophilic silanol moieties. However, organic sites (phenyl and methyl groups) form hydrophobic regions that block the hydrophilic base-soluble regions from the aqueous base solution, and inhibit film dissolution in the developer. ArF excimer-laser irradiation induced photo-oxidation breaks the phenyl-Si bond to form a polar oxide structure, such as silanol pendants, and makes the hydrophobic regions in the film smaller. That exposes the hydrophilic base-soluble regions to the aqueous base solution and promotes the film dissolution. In this scheme, the photo-oxidation acts as the switch that turns on the film dissolution.

\subsection{Resolution capability}

The result of a preliminary lithographic application of PMSQ as a resist for direct formation of hard-masks is shown in Fig. 5. The $0.2 \mu \mathrm{m}$-pitch-L/S PMSQ patterns were successfully obtained. The initial film thickness was $70 \mathrm{~nm}$, the film was pre-baked at $90{ }^{\circ} \mathrm{C}$ for $180 \mathrm{~s}$, and patterns could be formed by ArF excimerlaser exposure. We were also able to delineate a $0.2-\mu \mathrm{m}$ HSQ pattern, though the pattern was not well-defined because of the strong photo-absorption.

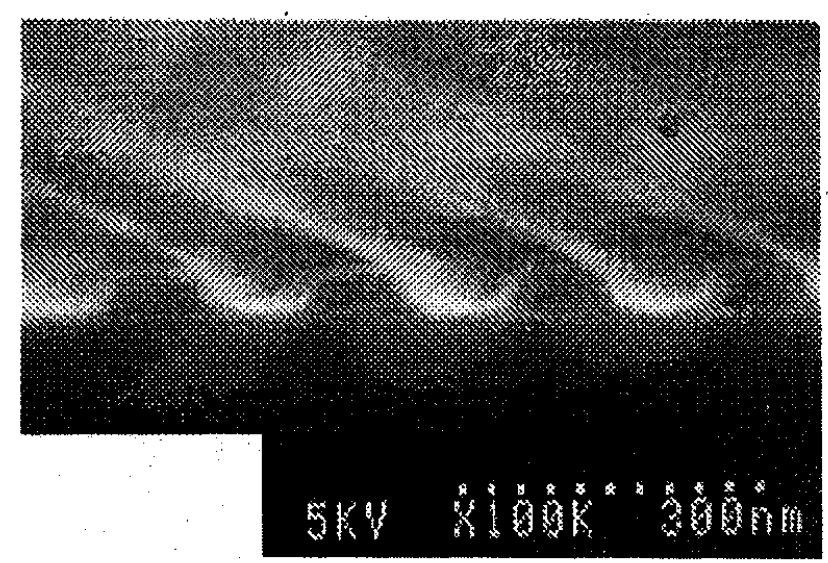

Figure 5 SEM micrograph of PMSQ-based resist patterns formed by using an ArF excimer laser with a holographic mask. 


\section{Summary}

Polyphenylmethyl silsesquioxane (PMSQ) showed positive tone characteristics when exposed by $\mathrm{ArF}$ excimer laser radiation followed by development with aqueous-base developer, while polyhydroxybenzyl-silsesquioxane (HSQ) exhibit negative tone characteristics. Infrared (IR) spectra and X-ray photoelectron spectrometry (XPS) suggests that a major mechanism of the pattern formation is the polarity change in PMSQ, while it is crosslinking in HSQ. PMSQ showed high resolution capability, resolving features as small as $0.1 \mu \mathrm{m}$.

\section{Acknowledgments}

We thank Dr. Masami Kouketsu for his assistance in the FT-IR measurement, Ms. Fumiko Yano for her assistance in the XPS measurement, and Dr. Shouichi Uchino for his comments and advice concerning the chemistry and purification of the materials. We also thank Dr. Hisashi Sugiyama for his advice on silsesquioxane polymer synthesis, Mr. Takeshi Furusawa for his comments on process feasibility, and Mr. Tsuneo Terasawa and Dr. Shinji Okazaki for his helpful discussion and continuous encouragement.

\section{References}

[1]a) Y.Kaimoto, K.Nozaki, S.Takechi, and N.Abe, "Alicyclic Polymer for ArF and KrF Excimer Resist Based on Chemical Amplification", Proc.SPIE 1672, pp66-73 (1992).

b) M.Takahashi, S.Takechi, Y.Kaimoto, I.Hanyu, N.Abe, and K.Nozaki, "Evaluation of chemically amplified resist based on adamantyl methacrylate for 193 nm lithography", Proc.SPIE 2438, pp422-432 (1995).

[2] M.Nakase, T.Naito, K.Asakawa, A.Hongu, N.Shida, and T.Ushirogouchi, "Chemically amplified ArF excimer laser resists using the absorption band shift method", Proc.SPIE 2438, pp445-454 (1995).

[3] R.D.Allen, G.M.Wallraff, R.A.DiPietro, D.C.Hofer, and R.R.Kunz, "193 nm Single Layer Positive Resists Building Etch Resistance Into a High Resolution Imaging System", Proc.SPIE 2438, pp474-485 (1995).

[4] M.A.Hartney, R.R.Kunz, D.J.Ehrlich, and D.C.Shaver, "Silylation processes for 193-nm excimer laser lithography", Proc.SPIE 1262, pp119-130 (1990).

[5] R.R.Kunz, M.W.Horn, R.B.Goodman, P.A.Bianconi, D.A.Smith, J.R.Eshelman, G.M.Wallraff, R.D.Miller, and E.J.Ginsburg, "Surface-Imaged Silicon Polymers for 193-nm Excimer Laser Lithography", Proc.SPIE 1672, pp385-393 (1992). 
[6] R.R.Kunz, M.W.Horn, P.A.Bianconi, D.A.Smith, and J.R.Eshelman, "Wetdeveloped bilayer resists for 193-nm excimer laser lithography", J.Vac.Sci.Technol.B10, pp2554-2559 (1992).

[7] T.W.Weideman, O.Joubert, A.M.Joshi, and R.1.Kostelak, "Applications of Plasma Polymerized Methylsilane as a Resist and Silicon Dioxide Precursor for 193 and 248 nm Lithography " , Proc.SPIE 2438, pp496-503 (1995).

[8] T.Morisawa and H.Fukuda, "Direct Formation of Hard Masks using Photooxidation of Polysilsesquioxane for ArF Excimer Laser Lithography", First Intn'l Symp. of ArF excimer laser lithography organized by SEMATECH, Aug. 1995 at Colorado Springs.

[9] H.Sugiyama, T.Inoue, A.Mizushima, and K.Nate, "Alkali-developable Organosilicon Positive Photoresist(OSPR)", Proc. SPIE 920, pp268-273 (1988).

[10] H.Watanabe, Y.Todokoro, and M.Inoue, "A Novel Silicon-containing Chemical Amplification Resist for Electron Beam Lithography", Microelectronic Eng. 13, pp69-72 (1991).

[11] N.Hayashi, T.Ueno, H.Shiraishi, T.Nishida, M.Toriumi, and S.Nonogaki, "A Silicon-containing Positive Photoresist Developable with Aqueous Alkaline Solution", ACS Symp. Series 346, pp211-223 (1987).

[12] H.Shiraishi, E.Fukuma, N.Hayashi, K.Tadano, and T.Ueno, "Insolubilization Mechanism of a Chemical Amplification Negative Resist System Utilizing an Acid-Catalyzed Silanol Condensation Reaction", Chem.Mater. 3, pp621-625 (1991). 